\title{
COVID19 admission risk tools should include multi-ethnic age structures, multimorbidity and deprivation metrics for air pollution, household overcrowding, housing quality and adult skills
}

\author{
Marina A. Soltan ( $\sim$ M.Soltan@bham.ac.uk) \\ University of Birmingham and University Hospitals Birmingham NHS Foundation Trust, Birmingham, UK \\ Justin Vamey \\ Birmingham City Council \\ Benjamin Sutton \\ University Hospitals Birmingham Foundation NHS Trust, Birmingham UK \\ Colin R. Melville \\ School of Medical Sciences, University of Manchester, Manchester, UK

\section{Sebastian T. Lugg} \\ University of Birmingham and University Hospitals Birmingham NHS Foundation Trust, Birmingham, UK \\ Dhruv Parekh \\ University of Birmingham and University Hospitals Birmingham NHS Foundation Trust, Birmingham, UK \\ Will Carroll \\ University Hospitals North Midlands, Newcastle Road, Stoke on Trent, UK \\ Davinder Dosanjh \\ University Hospitals Birmingham Foundation NHS Trust, Birmingham UK \\ David R. Thickett \\ University Hospitals Birmingham Foundation NHS Trust, Birmingham UK
}

\section{Research Article}

Keywords: BAME, ethnic minorities, COVID-19, ITU, Critical care, ethnicity, obesity, multimorbidity, household overcrowding, housing quality, air pollution, adult skills, education, language, multilobar pneumonia, outcome, mortality

Posted Date: April 6th, 2021

DOI: https://doi.org/10.21203/rs.3.rs-149632/v2

License: (c) (1) This work is licensed under a Creative Commons Attribution 4.0 International License. Read Full License

Version of Record: A version of this preprint was published at BMJ Open Respiratory Research on August 9th, 2021. See the published version at https://doi.org/10.1136/bmjresp-2021-000951. 


\section{Abstract}

Background-Ethnic minorities account for $34 \%$ of critically ill COVID-19 patients despite constituting $14 \%$ of the UK population. Internationally, researchers have called for studies to understand deterioration risk factors to inform clinical risk tool development.

Methods-Multi-centre cohort study of hospitalised COVID-19 patients ( $\mathrm{n}=3671$ ) exploring determinants of health, including Index of Multiple Deprivation (IMD) sub-indices, as risk factors for presentation, deterioration and mortality by ethnicity. Receiver operator characteristics were plotted for CURB65 and ISARIC4C by ethnicity and area under the curve (AUC) calculated.

Results-Ethnic minorities were admitted with higher Charlson Comorbidity Scores than age, sex and deprivation matched controls and from the highest IMD sub-indices of at least one deprivation form: Indoor Living Environment(LE), Outdoor LE, Adult Skills and Wider Barriers to Housing and Services. Admission from the highest sub-indices of these deprivation forms was associated with multilobar pneumonia on presentation and ITU admission. AUC did not exceed 0.7 for CURB65 or ISARIC4C among any ethnicity except ISARIC4C among Indian patients 0.83 (0.73-0.93). Ethnic minorities presenting with pneumonia and low CURB65(0-1) had higher mortality than Caucasians (22.6\% vs.9.4\%; $p<0.001)$; Africans were at highest risk (38.5\%; $p=0.006)$, followed by Caribbean (26.7\%; $p=0.008)$, Indian (23.1\%; $p=0.007)$ and Pakistani $(21.2 \% ; p=0.004)$.

Conclusions-Ethnic minorities exhibit higher multimorbidity despite younger age structures and disproportionate exposure to unscored risk factors: obesity and deprivation. Household overcrowding, air pollution, housing quality and adult skills deprivation are associated with multi-lobar pneumonia on presentation and ITU admission which are mortality risk factors. Risk tools need to reflect risks predominantly affecting ethnic minorities.

\section{Introduction}

Ethnic minorities account for $34 \%$ of critically ill patients with SARS-CoV-2 infection (COVID-19) despite constituting $14 \%$ of the UK population according to the UK Office for National Statistics. (1) Effective triage at the point of admission to hospital is required to ensure that patients from all ethnic groups are risk stratified to the appropriate level of care. Internationally, researchers have called for studies to understand deterioration and mortality risk factors to inform clinical risk tool development (2).

Diagnostic and prognostication models are valuable for risk stratification at the point of admission; more than 232 models for COVID-19 have been put forward by the academic community (3). However, critical appraisal of these models has identified that candidate models are poorly reported, at high risk of bias and their risk stratification performance among individual ethnic groups has not been reported (4). It is therefore unclear how well these proposed models perform in practice to risk stratify individual ethnic minority groups and whether models sufficiently account for biological and socioenvironmental risk factors to which ethnic minorities are predominantly predisposed.

We aimed to address this knowledge gap by exploring determinants of health, including Index of Multiple Deprivation (IMD) sub-indices, as risk factors for presentation, deterioration and mortality by ethnicity and by evaluating the performance of two widely used prognostic models, CURB65 (5) and ISARIC4C (6), among hospitalised patients diagnosed with COVID-19 by ethnicity.

Traditional clinical training has reinforced that the unmodifiable risk factor of age predisposes to adverse outcomes with little regard to epidemiological multiethnic age structures. Ethnic minorities have younger age structures (7) which predispose to a lower risk scoring using current risk stratification tools. Furthermore, ethnic minorities more frequently exhibit obesity and higher multimorbidity despite presenting younger yet this risk profile is not considered in current risk stratification tools.

Moreover, ethnic minorities are more likely than Caucasians to be hospitalised with COVID-19 from the most deprived IMD regions. (8) UK data published by the Office for National Statistics (ONS) shows higher age-standardised mortality rates for COVID-19 in the most deprived IMD areas (3.1 deaths per 100,000 patients) compared to the least deprived (1.4 deaths per 100,000) between $1^{\text {st }}$ March 2020 and $31^{\text {st }}$ July 2020. (9) However, studies have not yet explored individual IMD sub-indices as risk factors for presentation with multilobar pneumonia, Intensive Therapy Unit (ITU) admission and completed hospitalised episode outcomes. The IMD incorporates seven weighted deprivation sub-domains: Income, Employment, Health, Crime, Barriers to Housing and Services (BHS), Living Environment (LE) and Education, Skills and Training (EST). (10) BHS, LE and EST sub-domains each have two sub-indices. BHS sub-indices include (a) Geographical barriers, an indicator of proximity to local services, and (b) Wider BHS which contains an indicator for household overcrowding. LE sub-indices include (a) Indoor LE which has an indicator for housing quality and (b) Outdoor LE which is an indicator for air pollution. EST sub-indices include: (a) Children and Younger People's education attainment and (b) Adult Skills which contains indicators for adult qualifications and English language proficiency. (11)

Understanding these biological, demographic and socioenvironmental risk factors is invaluable when it comes to evaluating the resilience of current risk stratification tools and informing the development of stratification tools which reflect risk factors to which ethnic minorities are potentially disproportionately predisposed.

\section{Methods}

\section{Design and setting}

A multi-centre cohort study of hospitalised COVID-19 patients ( $n=3671)$ was performed to explore social determinants of health, including IMD sub-indices, as risk factors for presentation with multilobar pneumonia, ITU admission and hospitalised outcomes. 


\section{Patient population}

COVID-19 patients (>16 years old) admitted to 4 hospitals across the West Midlands comprising one of the UK's largest trusts, University Hospitals of Birmingham (UHB), between $1^{\text {st }}$ February 2020 and $1^{\text {st }}$ September 2020 were included. Diagnosis was confirmed by PCR analysis of a combined nose and throat swab in accordance with Public Health England guidance. (12)

\section{Patient management}

See online supplement 1.

\section{Data collection and scoring analysis}

Hospital informatics data included: demographics (ethnicity, age, IMD), admission details, comorbidities, clinical metrics (observations, blood tests), imaging, ITU admission details and hospitalised episode outcomes. Chest X-rays were reported by radiologists within 12 hours of being undertaken.

IMD

IMD sub-domains and sub-indices are detailed above. The IMD categorises deprivation metrics by postcode on a scale of 1 to 10 (most to least deprived centiles nationally). Detailed descriptions of IMD metrics are published by the UK Ministry of Housing, Communities and Local Government. (11)

\section{Charlson Comorbidity Index (CCl)}

$\mathrm{CCl}$ is a validated tool quantifying comorbidity burden and corresponding 1 year mortality risk. (13)

\section{CURB65 and ISARIC4C}

See online supplement 2 for characteristics of studies describing CURB65 (14) (5) and ISARIC4C mortality models (15).

\section{Statistical Analysis}

Baseline characteristics were presented as mean and standard deviation (SD) for continuous variables and median and interquartile range (IQR) for nonparametric data. Normality was assessed by Shapiro-Wilk. For categorical and ordinal variables with non-parametric distribution, Fisher's exact test and Mann Whitney $\mathrm{U}$ test were used respectively for comparisons between two groups. Age and sex adjusted mortality were calculated by logistic regression analyses. Multivariate analysis to predict mortality was performed using stepwise logistic regression with conservative criteria for entry or exit from the model of 0.1 . Variables listed in table 5a were included in multivariate analysis. The Hosmer and Lemeshow goodness-of-fit test was performed to evaluate logistic regression model adequacy. Matched case-control analyses (1:1) were implemented to explore underlying multimorbidity among ethnic minorities; controls were Caucasians matched by age, gender and deprivation sub-indices. Performance of the CURB65 and ISARIC 4C tools among individual ethnic groups were assessed using receiver operating characteristic curves (AUROC). Statistical analyses were carried out using SPSS V.24.

\section{Ethics statement}

Ethical approval was obtained by the Health Research Authority: REC reference 21/HRA/1299. The study was registered by the UHB Research and Development department - RRK7305.

Table 1-A table showing participant characteristics including: age, gender, ethnicity, ITU admission, mortality and discharge 


\begin{tabular}{|c|c|c|c|c|c|c|c|c|}
\hline & $\begin{array}{l}\text { All study } \\
\text { COVID-19- } \\
\text { positive } \\
\text { patients } \\
\text { median age } \\
\text { (IQR) }\end{array}$ & $\begin{array}{l}\text { All COVID- } \\
\text { 19-positive } \\
\text { patients } \\
\text { n, (\% of } \\
\text { column } \\
\text { total) }\end{array}$ & $\begin{array}{l}\text { COVID-19-positive patients } \\
\text { with Radiological Changes } \\
\text { of Pneumonia } \\
\text { n, (\% of row total) }\end{array}$ & $\begin{array}{l}\text { COVID-19 positive patients } \\
\text { with Radiological Changes of } \\
\text { multilobar pneumonia } \\
\text { n, ( } \% \text { of row total) }\end{array}$ & $\begin{array}{l}\text { COVID-19-positive patients } \\
\text { without Radiological } \\
\text { Changes of Pneumonia } \\
\mathrm{n} \text {, (\% of row total) }\end{array}$ & $\begin{array}{l}\text { ITU } \\
\text { admission } \\
\text { n, ( } \% \text { of } \\
\text { row total) }\end{array}$ & $\begin{array}{l}\text { Discharge } \\
\text { n, (\% of } \\
\text { row total) }\end{array}$ & $\begin{array}{l}\text { Mortality } \\
\mathrm{n},(\% \text { of } \\
\text { row } \\
\text { total) }\end{array}$ \\
\hline $\mathbf{N}$ & & 2646 & $1667(63.0)$ & 1307 (49.4) & 979 (37.0) & $\begin{array}{l}310 \\
(11.7)\end{array}$ & $\begin{array}{l}1771 \\
(66.9)\end{array}$ & $\begin{array}{l}875 \\
(33.1)\end{array}$ \\
\hline $\begin{array}{l}\text { Age, } \\
\text { median } \\
\text { (IQR) }\end{array}$ & & $76.0(24.0)$ & 70.8 (16.5) & 69.4 (16.6) & $73.7(17.7)$ & $\begin{array}{l}58.5 \\
(12.5)\end{array}$ & $\begin{array}{l}68.8 \\
(18.0)\end{array}$ & $\begin{array}{l}78.1 \\
(12.8)\end{array}$ \\
\hline \multicolumn{9}{|l|}{ Gender } \\
\hline Male & $73.0(24.0)$ & 1449 (54.8) & $970(66.9)$ & 775 (53.5) & 479 (33.1) & $\begin{array}{l}220 \\
(15.2)\end{array}$ & $\begin{array}{l}921 \\
(63.6)\end{array}$ & $\begin{array}{l}528 \\
(36.4)\end{array}$ \\
\hline Female & $79.0(23.0)$ & $1197(45.2)$ & $697(58.2)$ & $532(44.4)$ & $500(41.8)$ & $90(7.5)$ & $\begin{array}{l}802 \\
(67.0) \\
\end{array}$ & $\begin{array}{l}347 \\
(29.0) \\
\end{array}$ \\
\hline \multicolumn{9}{|l|}{ Ethnicity } \\
\hline Caucasian & 79.0 (19) & 1917 (72.4) & 1123 (58.6) & $831(43.3)$ & $794(41.4)$ & $\begin{array}{l}161 \\
(8.4) \\
\end{array}$ & $\begin{array}{l}1242 \\
(64.8) \\
\end{array}$ & $\begin{array}{l}675 \\
(35.2)\end{array}$ \\
\hline Indian & $63.0(23.5)$ & $93(3.5)$ & 77 (82.8) & 66 (71.0) & $16(17.2)$ & $21(22.6)$ & $67(72.0)$ & $\begin{array}{l}26 \\
(28.0) \\
\end{array}$ \\
\hline Pakistani & $62.0(29.0)$ & $326(12.3)$ & $245(75.2)$ & $216(66.3)$ & $81(24.8)$ & $66(20.2)$ & $\begin{array}{l}227 \\
(69.6) \\
\end{array}$ & $\begin{array}{l}99 \\
(30.4)\end{array}$ \\
\hline Caribbean & $73.0(28.0)$ & $105(4.0)$ & $69(65.7)$ & $58(55.2)$ & $36(34.3)$ & $10(9.5)$ & $73(69.5)$ & $\begin{array}{l}32 \\
(30.5)\end{array}$ \\
\hline African & $56.0(17.75)$ & $26(<1)$ & $22(84.6)$ & $19(73.1)$ & $4(15.4)$ & $8(30.8)$ & $16(61.5)$ & $\begin{array}{l}10 \\
(38.5) \\
\end{array}$ \\
\hline \begin{tabular}{l}
\multicolumn{1}{c}{ Any } \\
other ethnic \\
group
\end{tabular} & $56.0(24.0)$ & $111(4.2)$ & $83(74.7)$ & 76 (68.5) & $28(25.2)$ & 31 (27.9) & 95 (85.6) & $\begin{array}{l}16 \\
(14.4)\end{array}$ \\
\hline Chinese & $54.5(32.5)$ & $16(<1)$ & $12(75.0)$ & $12(75.0)$ & $4(25.0)$ & $6(37.5)$ & 13 (81.3) & $3(18.8)$ \\
\hline Bangladeshi & $45.0(38.0)$ & $11(<1)$ & $6(54.5)$ & $6(54.5)$ & $5(45.5)$ & $2(18.2)$ & $9(81.8)$ & $2(18.2)$ \\
\hline Mixed & $67.5(32.0)$ & $22(<1)$ & 15 (68.2) & $11(50.0)$ & 7 (31.8) & $2(9.1)$ & $14(63.6)$ & $8(36.4)$ \\
\hline Unspecified & $57.0(28.0)$ & $19(<1)$ & $15(78.9)$ & $12(63.2)$ & $4(21.1)$ & $3(15.8)$ & $15(78.9)$ & $4(21.1)$ \\
\hline $\begin{array}{l}\text { ITU } \\
\text { admission }\end{array}$ & $60.00(18.00)$ & $310(11.7)$ & $272(87.7)$ & $249(80.3)$ & 38 (12.3) & & & \\
\hline Discharge & $80.00(17.00)$ & 1771 (66.9) & 1037 (58.6) & $797(45.0)$ & $734(41.4)$ & & & \\
\hline Mortality & $72.0(28.0)$ & 875 (33.1) & $630(72.0)$ & $510(58.3)$ & 245 (28.0) & & & \\
\hline
\end{tabular}

\section{Results}

\section{Included participants}

3671 consecutive patients were assessed for inclusion. Figure 1 shows the CONSORT diagram.

\section{Study population}

\section{Age and sex}

The study population is outlined in table 1. Males (54.8\%) were hospitalised more than females (45.2\%). The median age of all patients was 76.0 (24.0). Ethnic minorities were more likely to present age $<65$ (OR 4.85(4.02-5.84); $<<0.001)$ than Caucasians. Caribbean and Caucasian subgroups presented older (median age>65) whilst Indian, Pakistani, African, Chinese and Bangladeshi subgroups presented younger (median age $<65$ ); this is consistent with UK population age structures. (7)

\section{Comorbidities}

Comorbidities by ethnic subgroup are shown in table 2 and Online Supplement 3. CCl scores among each ethnic minority group were higher than Caucasian controls matched by age, sex and deprivation sub-indicies (table 2). The average number of comorbidities among African, Pakistani and Caribbean patients was higher than age and sex matched Caucasian controls (table 3). Ethnic minorities were more likely to exhibit a BMI ${ }^{3} 30$ (OR 1.64(1.36-1.98); $\left.\mathrm{p}=0.001\right)$.

\section{Deprivation: household overcrowding, adult skills, housing quality and air pollution}

Hospitalised admissions by deprivation sub-index are depicted in figure 2 and ITU admissions by deprivation sub-index are depicted in figure $\mathbf{3}$. The proportion of patients admitted to hospital from the highest (sub-indices 1 and 2 ) deprivation forms were as follows: Wider BHS (59.0\%), Adult Skills (43.6\%), Indoor LE (42.3\%) and Outdoor LE (56.5\%).

The proportions of ethnic minorities vs Caucasian patients hospitalised from the highest (sub-indices 1 and 2) deprivation forms was as follows: Wider BHS ( $81.7 \%$ vs $50.2 \%$ ), Adult Skills (65.8\% vs. $35.1 \%)$, Indoor LE (54.6\% vs. $37.5 \%)$ and Outdoor LE ( $81.5 \%$ vs. $46.9 \%)$. Ethnic minorities were more likely than Caucasians to be admitted from the aforementioned deprivation forms, present with multi-lobar pneumonia (OR 2.465(2.057-2.945); $<<0.001)$ and require ITU admission (OR 2.823(2.219-3.611); p<0.001) (Online Supplement 4). 
Patients were more likely to present with radiological multilobar pneumonia if domiciled from regions of highest deprivation (sub-indices 1 and 2): Wider BHS (OR 1.66(1.42-1.95); $p=0.049)$, Indoor LE (OR 1.54(1.31-1.79); $p<0.0001)$, Outdoor LE (OR 1.76(1.51-2.06); $p<0.001$ ) and Adult Skills (OR 1.42(1.14-1.83); $\mathrm{p}=0.003$ ) compared with patients admitted from all other respective IMD sub-indices (fig. 4a). Patients presenting with multilobar pneumonia were more likely to require ITU admission (OR 4.93(3.68-6.60), p<0.000) and die (age and sex adjusted) (OR 2.20(1.84-2.63); p<0.000) (fig. 4a).

\section{Admission from highest deprivation sub-indices increases the risk of ITU admission}

Patients were more likely to be admitted to ITU if admitted from regions of highest deprivation (sub-indices 1 and 2): Wider BHS (OR 1.28(1.00-1.64); $p=0.048$ ), Indoor LE (OR 1.31(1.03-1.66); $p=0.028)$, Outdoor LE (OR 1.49(1.16-1.90); $p=0.002$ ) and Adult Skills (OR 1.44(1.14-1.83); $p=0.002)$ compared with patients admitted from all other respective IMD sub-indices (fig. 4b). Age and sex adjusted mortality was higher among patients admitted to ITU (OR 3.51(2.64-4.66); $p<0.000)$ (fig. 4b).

Table 2- A table representing underlying comorbidities and multimorbidity among hospitalised COVID-19 positive patients by ethnic subgroup: disaggregating ethnic minorities

\begin{tabular}{|c|c|c|c|c|c|c|c|c|c|c|c|}
\hline & $\begin{array}{l}\text { All COVID-19-positive } \\
\text { patients }\end{array}$ & Caucasian & $\begin{array}{l}\text { Ethnic } \\
\text { minorities }\end{array}$ & Pakistani & Indian & Caribbean & African & Chinese & Bangladeshi & Mixed & $\begin{array}{l}\text { Any other ethnic } \\
\text { group }\end{array}$ \\
\hline & 2646 & $\begin{array}{l}1917 \\
(72.4)\end{array}$ & $710(26.8)$ & $\begin{array}{l}326 \\
(12.3)\end{array}$ & $93(3.5)$ & $105(4.0)$ & $26(<1)$ & $16(<1)$ & $11(<1)$ & $\begin{array}{l}22 \\
(<1)\end{array}$ & $111(4.2)$ \\
\hline & \multicolumn{11}{|c|}{ Cardiovascular ( $n, \%$ of column) } \\
\hline HTN & $1030(38.9)$ & $736(38.4)$ & $266(37.4)$ & $\begin{array}{l}130 \\
(39.9)\end{array}$ & $\begin{array}{l}43 \\
(46.2)\end{array}$ & $53(50.5)$ & $9(34.6)$ & $5(31.3)$ & $2(18.2)$ & $\begin{array}{l}6 \\
(27.3)\end{array}$ & $40(36.0)$ \\
\hline IHD & $433(16.4)$ & 317 (16.5) & $113(15.9)$ & $53(16.3)$ & $\begin{array}{l}14 \\
(15.1)\end{array}$ & $15(14.3)$ & $5(19.2)$ & $0(0)$ & $0(0)$ & $\begin{array}{l}20 \\
(90.9)\end{array}$ & $6(5.4)$ \\
\hline Hypercholesterolaemia & $206(7.8)$ & $145(7.6)$ & $56(7.9)$ & $33(10.1)$ & $4(4.3)$ & $6(5.7)$ & $3(11.5)$ & $2(12.5)$ & $1(9.1)$ & $\begin{array}{l}6 \\
(27.3) \\
\end{array}$ & $1(0.9)$ \\
\hline $\mathrm{CCF}$ & 208 & $166(8.7)$ & $42(5.9)$ & $24(7.4)$ & $5(5.4)$ & $6(5.7)$ & $0(0)$ & $0(0)$ & $0(0)$ & $\begin{array}{l}6 \\
(27.3)\end{array}$ & $1(0.9)$ \\
\hline $\begin{array}{l}\text { Peripheral Vascular } \\
\text { disease }\end{array}$ & $64(2.4)$ & $56(2.9)$ & $8(1.1)$ & $2(0.6)$ & $1(1.1)$ & $5(4.8)$ & $0(0)$ & $0(0)$ & $0(0)$ & $0(0)$ & $0(0)$ \\
\hline \multirow[t]{2}{*}{ Stroke } & $215(8.1)$ & $170(8.9)$ & $44(6.2)$ & $12(3.7)$ & $3(3.2)$ & $15(14.3)$ & $2(7.7)$ & $1(6.3)$ & $1(9.1)$ & $\begin{array}{l}7 \\
(31.8) \\
\end{array}$ & $3(2.7)$ \\
\hline & \multicolumn{10}{|l|}{ Respiratory ( $\mathrm{n}, \%$ of column) } & \\
\hline COPD & $269(10.2)$ & $202(10.5)$ & $67(9.4)$ & $28(8.6)$ & $9(9.7)$ & $8(7.6)$ & $3(11.5)$ & $2(12.5)$ & $0(0)$ & $\begin{array}{l}12 \\
(54.5)\end{array}$ & $3(2.7)$ \\
\hline ILD & $53(2.0)$ & $42(2.2)$ & $11(1.5)$ & $4(1.2)$ & $1(1.1)$ & $2(1.9)$ & $1(3.8)$ & $1(6.3)$ & $0(0)$ & $2(9.1)$ & $0(0)$ \\
\hline OSA & $51(1.2)$ & $38(2.0)$ & $13(1.8)$ & $5(1.5)$ & $2(2.2)$ & $2(1.9)$ & $0(0)$ & $1(6.3)$ & $0(0)$ & $\begin{array}{l}3 \\
(13.6)\end{array}$ & $0(0)$ \\
\hline \multirow[t]{2}{*}{ Bronchiectasis } & $32(1.2)$ & $27(1.4)$ & $5(0.7)$ & $3(0.9)$ & $0(0)$ & $0(0)$ & $1(3.8)$ & $0(0)$ & $0(0)$ & $0(0)$ & $0(0)$ \\
\hline & \multicolumn{11}{|l|}{ Renal ( $n, \%$ of column) } \\
\hline \multirow[t]{2}{*}{ CKD } & $355(13.4)$ & $262(13.7)$ & $93(13.1)$ & $39(12.0)$ & $\begin{array}{l}14 \\
(15.1)\end{array}$ & $11(10.5)$ & $3(11.5)$ & $1(6.3)$ & $2(18.2)$ & $\begin{array}{l}15 \\
(68.2)\end{array}$ & $6(5.4)$ \\
\hline & \multicolumn{11}{|c|}{ Endocrinology (n, \% of column) } \\
\hline T1DM & $26(1.0)$ & $23(1.2)$ & $3(0.4)$ & $2(0.6)$ & $0(0)$ & $0(0)$ & $0(0)$ & $1(6.3)$ & $0(0)$ & $0(0)$ & $0(0)$ \\
\hline T2DM & $713(26.9)$ & $517(27.0)$ & $196(27.6)$ & $81(24.8)$ & $\begin{array}{l}29 \\
(31.2)\end{array}$ & $26(24.8)$ & $5(19.2)$ & $2(12.5)$ & $2(18.2)$ & $\begin{array}{l}6 \\
(27.3)\end{array}$ & $8(7.2)$ \\
\hline Vitamin $\mathrm{D}<20$ & $56(2.1)$ & $38(2.0)$ & $18(2.5)$ & $7(2.1)$ & $4(4.3)$ & $3(2.9)$ & $2(7.7)$ & $0(0)$ & $0(0)$ & $2(9.1)$ & $0(0)$ \\
\hline \multirow[t]{2}{*}{ BMI $>30$} & $742(28.0)$ & $481(25.1)$ & $261(36.8)$ & $\begin{array}{l}136 \\
(41.7) \\
\end{array}$ & $\begin{array}{l}22 \\
(23.7) \\
\end{array}$ & $34(32.4)$ & $\begin{array}{l}10 \\
(38.5)\end{array}$ & $5(31.3)$ & $2(18.2)$ & $\begin{array}{l}8 \\
(36.4) \\
\end{array}$ & 35 (31.5) \\
\hline & \multicolumn{11}{|l|}{ Hepatobiliary ( $\mathrm{n}, \%$ of column) } \\
\hline Hepatitis & $14(0.5)$ & $4(0.2)$ & $10(1.4)$ & $4(1.2)$ & $4(4.3)$ & $1(1.0)$ & $2(2.7)$ & $1(6.3)$ & $0(0)$ & $1(4.5)$ & $1(0.9)$ \\
\hline Cirrhosis & $22(0.8)$ & $21(1.1)$ & $1(0.1)$ & $0(0)$ & $0(0)$ & $0(0)$ & $0(0)$ & $0(0)$ & $0(0)$ & $0(0)$ & $1(0.9)$ \\
\hline Peptic Ulcer Disease & $35(1.3)$ & $30(1.6)$ & $5(0.7)$ & $2(0.6)$ & $1(1.1)$ & $0(0)$ & $0(0)$ & $0(0)$ & $0(0)$ & $1(4.5)$ & $0(0)$ \\
\hline \multirow[t]{2}{*}{ Variceal GI bleed } & $12(0.5)$ & $10(0.5)$ & $2(0.3)$ & $0(0)$ & $0(0)$ & $1(1.0)$ & $0(0)$ & $0(0)$ & $0(0)$ & $1(4.5)$ & $0(0)$ \\
\hline & \multicolumn{11}{|c|}{ Rheumatology (n, \% of column) } \\
\hline $\begin{array}{l}\text { Connective Tissue } \\
\text { Disease }\end{array}$ & $204(7.7)$ & $166(8.7)$ & $38(5.4)$ & $15(4.6)$ & $5(5.4)$ & $8(7.6)$ & $3(11.5)$ & $3(18.8)$ & $0(0)$ & $\begin{array}{l}4 \\
(18.2)\end{array}$ & $0(0)$ \\
\hline & & & & & & & & & & & \\
\hline & \multicolumn{11}{|c|}{ Multimorbidity ( $n, \%$ of column) } \\
\hline$>1$ comorbidity & $2042(81.4)$ & $\begin{array}{l}1555 \\
(84.7) \\
\end{array}$ & $472(66.5)$ & $\begin{array}{l}209 \\
(69.4)\end{array}$ & $\begin{array}{l}66 \\
(75.0)\end{array}$ & $87(85.3)$ & $\begin{array}{l}19 \\
(76.0)\end{array}$ & $\begin{array}{l}12 \\
(80.0)\end{array}$ & $5(45.5)$ & $\begin{array}{l}14 \\
(77.8)\end{array}$ & $74(66.7)$ \\
\hline 4 or more comorbidities & $791(31.6)$ & $641(31.6)$ & $147(20.7)$ & $68(22.6)$ & $\begin{array}{l}16 \\
(18.2)\end{array}$ & $31(30.4)$ & $6(24.0)$ & $1(6.7)$ & $0(0)$ & $\begin{array}{l}3 \\
(16.7)\end{array}$ & $25(22.5)$ \\
\hline
\end{tabular}

Table 3-A table representing Charlson Comorbidity Index (CCI) Scores among patients of ethnic minorities in comparison with matched controls by: a) Age and Gender, b) Age, Gender and Outdoor LE deprivation, c) Age Gender and Indoor LE deprivation, d) Age, Gender and Adult Skills deprivation. 


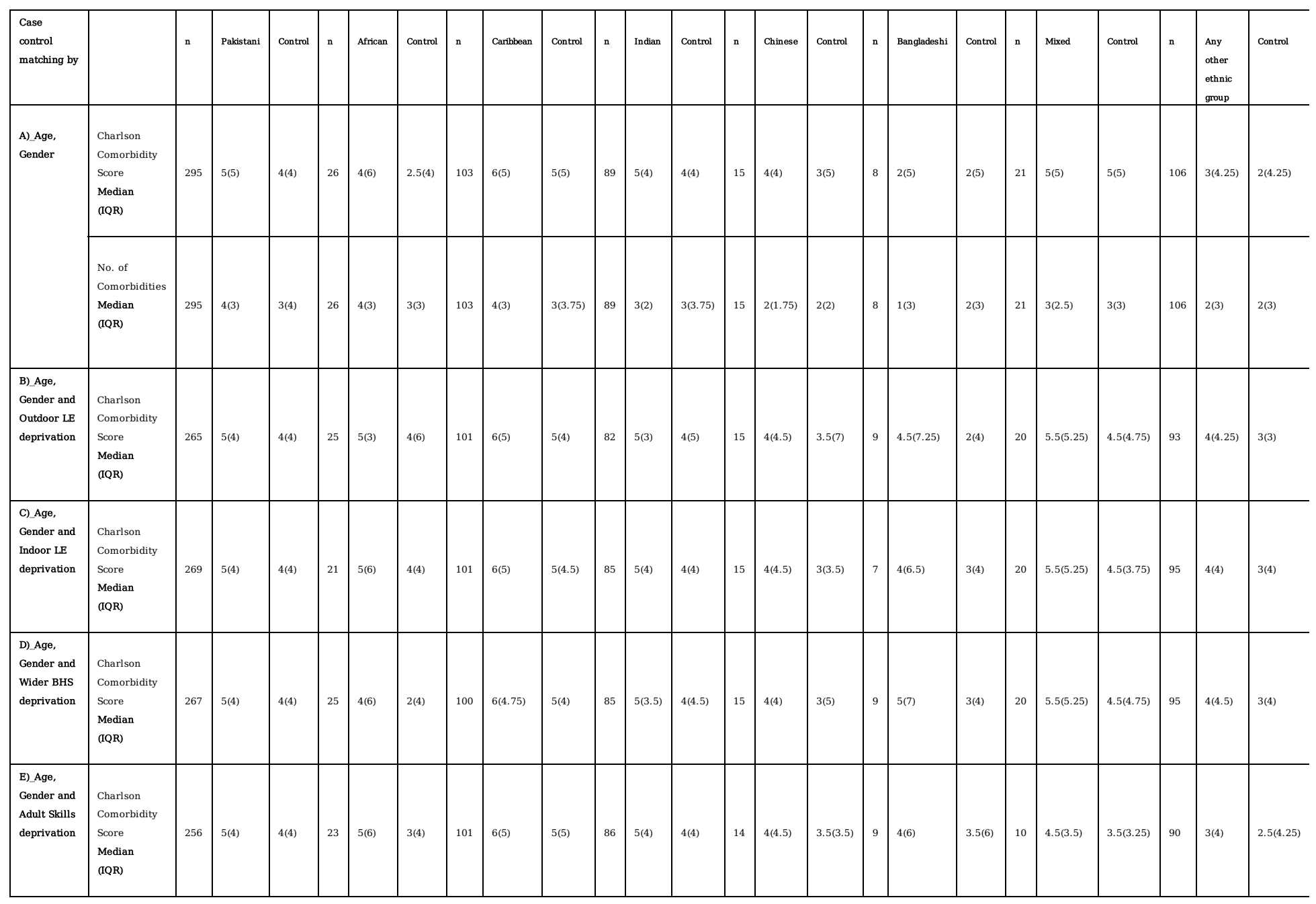

Ethnic minorities: IMD sub-indices, presentation and ITU admission

Table 4 depicts the proportion of admissions from the respective deprivation forms by ethnicity.

Table 4-Admissions by highest deprivation: BHS, Wider BHS, LE, Indoor LE, Outdoor LE and Adult Skills

\begin{tabular}{|c|c|c|c|c|c|c|c|}
\hline & $\mathbf{N}$ & $\begin{array}{l}\text { Admission from } \\
\text { regions of highest } \\
\text { BHS deprivation } \\
\end{array}$ & $\begin{array}{l}\text { Admission from regions } \\
\text { of highest Wider BHS } \\
\text { deprivation }\end{array}$ & $\begin{array}{l}\text { Admission from } \\
\text { regions of highest } \\
\text { LE deprivation }\end{array}$ & $\begin{array}{l}\text { Admission from regions } \\
\text { of highest Indoor LE } \\
\text { deprivation }\end{array}$ & $\begin{array}{l}\text { Admission from regions } \\
\text { of highest Outdoor LE } \\
\text { deprivation }\end{array}$ & $\begin{array}{l}\text { Admission from regions } \\
\text { of highest Adult Skills } \\
\text { deprivation }\end{array}$ \\
\hline \multicolumn{8}{|c|}{ Ethnic group, $\mathbf{n}$ (\% of ethnic group) } \\
\hline Caucasian & 1917 & $566(29.5)$ & $963(50.2)$ & $815(42.4)$ & $719(37.5)$ & $900(46.9)$ & $673(35.1)$ \\
\hline $\begin{array}{r}\text { Ethnic } \\
\text { minorities }\end{array}$ & 710 & $442(62.3)$ & $580(81.7)$ & $506(71.3)$ & $388(54.6)$ & $579(81.5)$ & $467(65.8)$ \\
\hline Indian & 93 & $34(36.6)$ & $54(58.1)$ & $48(51.6)$ & $35(37.6)$ & $65(69.9)$ & $34(36.6)$ \\
\hline Pakistani & 326 & $250(76.7)$ & $293(89.9)$ & $263(80.7)$ & $202(62.0)$ & $290(89.0)$ & $266(81.6)$ \\
\hline Caribbean & 105 & $59(56.2)$ & $88(83.8)$ & $76(72.4)$ & $55(52.4)$ & $89(84.8)$ & $53(50.5)$ \\
\hline African & 26 & $15(57.7)$ & $21(80.8)$ & $18(69.2)$ & $13(50)$ & $19(73.1)$ & $20(76.9)$ \\
\hline Chinese & 16 & $4(25.0)$ & $13(81.3)$ & $10(62.5)$ & $9(56.3)$ & $11(68.8)$ & $7(43.8)$ \\
\hline Bangladeshi & 11 & $8(72.7)$ & $9(81.8)$ & $8(72.7)$ & $6(54.5)$ & $9(81.8)$ & $7(63.6)$ \\
\hline Mixed & 22 & $13(59.1)$ & $17(77.3)$ & $12(54.5)$ & $8(36.4)$ & $14(63.6)$ & $16(72.7)$ \\
\hline $\begin{array}{l}\text { Any } \\
\text { other ethnic } \\
\text { group }\end{array}$ & 111 & $59(53.2)$ & 85 (76.6) & $71(64.0)$ & $60(54.1)$ & $82(73.9)$ & 64 (57.7) \\
\hline Unspecified & 19 & $13(68.4)$ & $18(94.7)$ & $14(73.7)$ & $11(57.9)$ & $16(84.2)$ & $13(68.4)$ \\
\hline Total & 2646 & $1021(38.6)$ & $1561(59.0)$ & $1335(50.5)$ & $1118(42.3)$ & $1495(56.5)$ & $1153(43.6)$ \\
\hline
\end{tabular}

$\underline{\text { Indian }}$ 
Indian patients were more likely than Caucasians to be admitted from regions of highest (sub-indices 1 and 2) Outdoor LE deprivation (OR 2.62(1.68-4.07); $p<0.001$ ), present with multilobar pneumonia (OR 3.20(2.03 to 5.03); $p<0.001$ ) and require ITU admission (OR 3.18(1.91 to 5.31); $p<0.001)$ (fig. 6a).

\section{$\underline{\text { Pakistani }}$}

Pakistani patients were more likely than Caucasians to be admitted from regions of highest deprivation (sub-indices 1 and 2): Wider BHS (OR 8.80(6.1312.76); $p<0.001$ ), Outdoor LE (OR 9.10(6.39-13.08); $p<0.001$ ), Indoor LE (OR 2.71(2.14-3.46); $p<0.001)$, Adult Skills (OR 8.20(6.10-11.02); $p<0.001$ ), present with multilobar pneumonia (OR 2.57(2.01-3.28); $p<0.001$ ) and require ITU admission (OR 2.77(2.02-3.79); $p<0.000)$ (fig. 6b).

\section{African}

Africans were more likely than Caucasians to be admitted from regions of highest deprivation (sub-indices 1 and 2): Wider BHS (OR 4.16(1.58-10.17); $p=0.002$ ), Outdoor LE (OR 3.07(1.31-7.72); $p=0.009)$, Adult Skills (OR 6.16(2.50-14.57); $p<0.001)$, present with multilobar pneumonia (OR 3.55(1.51-8.92); $\mathrm{p}=0.004)$ and require ITU admission (OR 4.85(2.08-11.32); $p<0.000)$ (fig. 6c).

\section{Caribbean}

Caribbean patients were more likely than Caucasians to be admitted from regions of highest deprivation (sub-indices 1 and 2): Wider BHS (OR 5.13(3.04-8.65); p<0.001), Indoor LE (OR 1.83(1.25-2.71); $p=0.003)$, Outdoor LE (OR 6.29(3.66-11.05); $p<0.001)$, Adult Skills (OR 1.88(1.28-2.78); $p=0.002)$ and present with multilobar pneumonia (OR 1.61(1.09-2.40); $p=0.020)$ (fig. 6d). Caribbean patients were not more likely to require ITU admission ( $>0.05)$.

\section{Chinese}

Chinese patients were more likely than Caucasians to be admitted from regions of highest deprivation (sub-indices 1 and 2): Wider BHS (OR 4.29(1.27-14.20); $p=0.021$ ), present with multilobar pneumonia (OR 3.92(1.26-11.16); $p=0.020)$ and require ITU admission (OR 6.54(2.35-18.24); $p<0.000)($ fig. 6e).

\section{Bangladeshi}

Bangladeshi patients were more likely than Caucasians to be admitted from regions of highest deprivation (sub-indices 1 and 2): Wider BHS (OR 4.46(1.1120.63); =0.037), Outdoor LE (OR 5.09(1.27-23.53; p<0.001) and Adult Skills (OR 3.24(1.04-9.91); p=0.048) although they were not more likely to present with multilobar pneumonia or require ITU admission (fig. 6f).

Mixed

Mixed ethnicity patients were more likely than Caucasians to be admitted from regions of highest deprivation (sub-indices 1 and 2): Wider BHS (OR 3.37(1.308.37); $p=0.016$ ) and Adult Skills (OR 4.93(2.01-12.07); $p=0.001$ ) although they were not more likely to present with multilobar pneumonia or require ITU admission (fig. 6g).

\section{$\underline{\text { Any other non-Caucasian ethnic group }}$}

Patients of any other non-Caucasian ethnicity were more likely than Caucasians to be admitted from regions of highest deprivation (sub-indices 1 and 2): Wider BHS (OR 3.24(2.07-5.06); p<0.001), Indoor LE (OR 1.96(1.34-2.85); p<0.001), Outdoor LE (OR 3.20(2.08-4.95); p<0.001), Adult Skills (OR 2.52(1.71-3.71); $\mathrm{p}<0.001)$, present with multilobar pneumonia (OR 2.84(1.88-4.25); $\mathrm{p}<0.001)$ and require ITU admission (OR 3.82(2.43-6.01); $p<0.000)(\mathrm{fig} .6 \mathrm{~h})$.

\section{Risk factors for mortality}

Multivariate analysis including variables in table $5 \mathrm{a}$ and identified seven variables which were independently associated with mortality (table $5 \mathrm{~b}$ ): age, sex, obesity, cirrhosis, IHD, CCl score and presentation with multilobar pneumonia.

Table 5a - Univariate analysis to predict mortality 


\begin{tabular}{|c|c|c|c|c|}
\hline & Odds Ratio & 99\% CI Lower bound & 99\% CI Upper bound & p-value \\
\hline Age & 1.04 & 1.03 & 1.04 & $<0.000$ \\
\hline Male sex & 1.40 & 1.19 & 1.40 & $<0.000$ \\
\hline Obesity (BMI $\square$ 30) & 3.32 & 2.77 & 3.96 & $<0.001$ \\
\hline Hypertension & 1.23 & 1.04 & 1.45 & 0.018 \\
\hline Ischaemic Heart Disease & 1.34 & 1.08 & 1.66 & $<0.009$ \\
\hline Heart Failure & 1.38 & 1.03 & 1.84 & $<0.032$ \\
\hline Peripheral Vascular Disease & 1.81 & 1.10 & 2.95 & $<0.022$ \\
\hline COPD & 1.34 & 1.03 & 1.73 & $<0.034$ \\
\hline Type 2 Diabetes Mellitus & 1.21 & 1.01 & 1.45 & $<0.041$ \\
\hline Cirrhosis & 4.40 & 1.79 & 10.82 & $<0.0009$ \\
\hline Chronic Kidney Disease & 1.44 & 1.14 & 1.81 & $<0.002$ \\
\hline Charlson Comorbidity (CCI) Score & 1.19 & 1.16 & 1.23 & $<0.000$ \\
\hline Multilobar pneumonia & 2.13 & 1.77 & 2.57 & $<0.000$ \\
\hline Index of Multiple Deprivation & 0.88 & 0.75 & 1.04 & 0.126 \\
\hline Wider BHS deprivation & 0.92 & 0.78 & 1.08 & 0.305 \\
\hline Outdoor LE deprivation & 0.85 & 0.72 & 0.99 & 0.042 \\
\hline Indoor LE deprivation & 0.92 & 0.78 & 1.08 & 0.288 \\
\hline Adult Skills deprivation & 0.91 & 0.77 & 1.07 & 0.268 \\
\hline Ethnic minorities & 0.69 & 0.57 & 0.84 & $<0.000$ \\
\hline Pakistani & 1.34 & 1.01 & 1.77 & $<0.041$ \\
\hline African & 2.42 & 1.04 & 5.61 & $<0.040$ \\
\hline Carribean & 0.94 & 0.60 & 1.47 & 0.787 \\
\hline Indian & 0.92 & 0.56 & 1.50 & 0.726 \\
\hline Bangladeshi & 0.92 & 0.18 & 4.65 & 0.917 \\
\hline Chinese & 0.68 & 0.18 & 2.51 & 0.559 \\
\hline Mixed & 1.72 & 0.68 & 4.34 & 0.255 \\
\hline Any other ethnic group & 0.53 & 0.30 & 0.93 & 0.028 \\
\hline
\end{tabular}

Table $5 b$ - Multivariate analysis to predict mortality

\begin{tabular}{|l|l|l|l|l|}
\hline & Odds Ratio & 99\% CI Lower bound & 99\% CI upper bound & P value \\
\hline Age & 1.05 & 1.04 & 1.06 & $<0.000$ \\
\hline Male sex & 1.50 & 1.25 & 1.81 & $<0.000$ \\
\hline Charlson Comorbidity (CCI) Score & 1.11 & 1.06 & 1.16 & $<0.000$ \\
\hline Obesity (BMI 0 30) & 3.60 & 2.95 & 4.38 & $<0.000$ \\
\hline Ischaemic Heart Disease & 0.78 & 0.60 & 0.99 & 0.047 \\
\hline Cirrhosis & 9.72 & 3.47 & 27.17 & $<0.000$ \\
\hline Multilobar pneumonia & 1.89 & 1.56 & 2.28 & $<0.000$ \\
\hline
\end{tabular}

\section{Clinical risk stratification tools}

AUROC was used to test the performance of the CURB65 and ISARIC 4C scores in predicting in-hospital mortality by ethnic group. Highest AUROC curves were achieved by the ISARIC4C score for the prediction of in-hospital mortality among Indian patients $(0.83 ; 95 \% \mathrm{CI} 0.73-0.93)$. Area under the curve (AUC) did not exceed 0.7 for CURB65 or ISARIC4C among any of the other ethnic groups (Figure 7 and Online supplement 5).

Ethnic minorities with pneumonia and low CURB65 scores (0-1) had higher mortality than Caucasians (22.6\% vs.9.4\%; $p<0.001)$; in comparison to Caucasians, Africans were at highest risk 38.5\% (OR 6.05(2.13-18.89); $p=0.006)$, followed by Caribbean 26.7\% (OR 3.52(1.53-8.45); $p=0.008)$, Indian 23.1\% (OR 2.90(1.436.07); $p=0.007$ ) and Pakistani 21.2\% (OR 2.56(1.42-4.66); $p=0.004)$. Table 6 disaggregates CURB65 scores by ethnic subgroup.

Table 6- CURB65 scores among COVID-19 patients presenting with pneumonia by ethnic subgroup 


\begin{tabular}{|c|c|c|c|c|c|c|c|c|c|c|c|}
\hline & \multirow{2}{*}{$\begin{array}{l}\text { Number of patients } \\
\text { presenting with } \\
\text { pneumonia }\end{array}$} & \multirow{2}{*}{$\begin{array}{l}\text { Number of patients } \\
\text { with CURB } 65 \text { score } \\
\text { data }\end{array}$} & \multicolumn{3}{|c|}{ CURB65 score 0 - 1} & \multicolumn{3}{|c|}{ CURB65 score 2} & \multicolumn{3}{|c|}{ CURB65 scores 3 - 5} \\
\hline & & & $\begin{array}{l}\text { Total } \\
\text { no } \\
\mathrm{N}(\% \\
\text { of } \\
\text { total) }\end{array}$ & $\begin{array}{l}\text { Died } \\
\mathrm{N} \text { (\% of } \\
\text { patients with } \\
\text { CURB65 0-1) }\end{array}$ & $\begin{array}{l}\text { Discharged } \\
\mathrm{N} \text { (\% of } \\
\text { patients with } \\
\text { CURB65 0-1) }\end{array}$ & $\begin{array}{l}\text { Total } \\
\text { no } \\
\mathrm{N}(\% \\
\text { of } \\
\text { total) }\end{array}$ & $\begin{array}{l}\text { Died } \\
\mathrm{N} \text { (\% of } \\
\text { patients with } \\
\text { CURB65 2) }\end{array}$ & $\begin{array}{l}\text { Discharged } \\
\mathrm{N} \text { (\% of } \\
\text { patients with } \\
\text { CURB65 2) }\end{array}$ & $\begin{array}{l}\text { Total no } \\
\mathrm{N} \text { (\% of } \\
\text { total) }\end{array}$ & $\begin{array}{l}\text { Died } \\
\mathrm{N} \text { (\% of } \\
\text { patients with } \\
\text { CURB65 3-5) }\end{array}$ & $\begin{array}{l}\text { Discharged } \\
\mathrm{N} \text { (\% of } \\
\text { patients with } \\
\text { CURB65 3-5) }\end{array}$ \\
\hline Caucasian & 1123 & 1110 & $\begin{array}{l}427 \\
(38.5) \\
\end{array}$ & $40(9.4)$ & 387 (90.6) & $\begin{array}{l}322 \\
(29.0)\end{array}$ & 59 (18.3) & $263(81.7)$ & $361(32.5)$ & $110(30.5)$ & $251(69.5)$ \\
\hline $\begin{array}{l}\text { Ethnic } \\
\text { minorities }\end{array}$ & 529 & 419 & $\begin{array}{l}252 \\
(60.1) \\
\end{array}$ & $57(22.6)$ & $196(77.8)$ & $\begin{array}{l}92 \\
(22.0) \\
\end{array}$ & $16(17.4)$ & 77 (83.7) & $76(18.1)$ & $16(21.1)$ & $58(76.3)$ \\
\hline Pakistani & 245 & 163 & $\begin{array}{l}85 \\
(52.1) \\
\end{array}$ & $18(21.2)$ & $68(81.9)$ & $\begin{array}{l}46 \\
(28.2) \\
\end{array}$ & $8(17.4)$ & $39(84.8)$ & $32(19.6)$ & $9(28.1)$ & 21 (61.8) \\
\hline Indian & 77 & 73 & $\begin{array}{l}52 \\
(71.2)\end{array}$ & $12(23.1)$ & $40(76.9)$ & $\begin{array}{l}9 \\
(12.3) \\
\end{array}$ & $1(11.1)$ & $8(88.9)$ & $12(2.7)$ & $1(8.3)$ & $11(91.7)$ \\
\hline Caribbean & 69 & 62 & $\begin{array}{l}30 \\
(48.4)\end{array}$ & $8(26.7)$ & $22(73.3)$ & $\begin{array}{l}19 \\
(30.6)\end{array}$ & $5(26.3)$ & $14(73.7)$ & $13(21.0)$ & $4(30.8)$ & $9(69.2)$ \\
\hline African & 22 & 13 & $\begin{array}{l}13 \\
(100) \\
\end{array}$ & 5 (38.5) & $8(61.5)$ & $0(0)$ & $0(0)$ & $0(0)$ & $0(0)$ & $0(0.0)$ & $0(0.0)$ \\
\hline Chinese & 12 & 12 & $\begin{array}{l}6 \\
(50.0)\end{array}$ & 1 (16.7) & $5(83.3)$ & $\begin{array}{l}3 \\
(25.0)\end{array}$ & $0(0)$ & $3(100)$ & $3(25.0)$ & $0(0.0)$ & $3(100)$ \\
\hline Bangladeshi & 6 & 5 & $\begin{array}{l}5 \\
(100) \\
\end{array}$ & $0(0.0)$ & $5(100)$ & $0(0)$ & $0(0)$ & $0(0)$ & $0(0)$ & $0(0.0)$ & $0(0.0)$ \\
\hline Mixed & 15 & 12 & $\begin{array}{l}8 \\
(76.9) \\
\end{array}$ & $2(25.0)$ & $6(75.0)$ & $\begin{array}{l}2 \\
(7.7) \\
\end{array}$ & $1(50.0)$ & $1(50.0)$ & $2(15.4)$ & $0(0.0)$ & $2(100)$ \\
\hline $\begin{array}{l}\text { Any other } \\
\text { ethnic } \\
\text { group }\end{array}$ & 83 & 79 & $\begin{array}{l}51 \\
(64.6)\end{array}$ & $9(17.6)$ & $42(82.4)$ & $\begin{array}{l}14 \\
(17.7)\end{array}$ & $2(14.3)$ & $12(85.7)$ & $14(17.7)$ & $2(14.3)$ & $12(85.7)$ \\
\hline Unspecified & 15 & 15 & $\begin{array}{l}9 \\
(60.0) \\
\end{array}$ & $0(0.0)$ & $9(100)$ & $\begin{array}{l}5 \\
(33.3) \\
\end{array}$ & $1(20.0)$ & $4(80.0)$ & $1(6.7)$ & $0(0)$ & $1(100)$ \\
\hline Total & 1667 & 1544 & & & & & & & & & \\
\hline
\end{tabular}

\section{Discussion}

Ethnic minorities are more likely to be hospitalised with COVID19 from regions of highest deprivation; admission from regions of highest Indoor LE deprivation, Outdoor LE deprivation, Wider BHS deprivation and Adult Skills deprivation are associated with multilobar pneumonia on presentation and ITU admission which are mortality risk factors. Deprivation metrics are not incorporated within current clinical risk stratification tools for hospitalised patients with COVID19 or pneumonia. This may explain the higher ITU admissions among ethnic minorities reported by ICNARC (1) and ONS data reporting higher age standardised mortality rates among patients in the most deprived IMD areas. (9)

Furthermore, ethnic minorities exhibit younger age-structures which results in underscoring using the 232 diagnostic or prognostic clinical risk stratification tools identified in a relevant systematic review. (3) Moreover, despite presenting with younger-age structures, ethnic minorities present with higher CCI scores and a higher incidence of obesity yet neither factor are accounted for in commonly used COVID-19 admission clinical risk stratification tools. Hospitalised COVID19 patients with underlying obesity, hypertension, IHD, heart failure, CKD, PVD, T2DM, cirrhosis and CKD are at increased risk of mortality. Clusters of disease are known to increase mortality (16) and affect ethnic groups differentially (17) yet current COVID19 admission clinical risk tools do not account for clusters of disease or $\mathrm{CCl}$ scores despite warning from the UK's Chief Medical Officer regarding rising multimorbidity (18) and the resultant challenges for acute and long-term care provision (21-22).

The oversight of scoring biological, demographic and socioenvironmental risk factors to which ethnic minorities are predominantly predisposed results in potential underscoring and triage to an inappropriate level of care whilst clinicians are left falsely reassured regarding the severity of presentation and risk of deterioration.

It is perhaps therefore not surprising therefore that the AUROC analyses demonstrated generally poor performance of the CURB65 (5) and ISARIC 4C (6) risk stratification tools among individual ethnic groups hospitalised with COVID19. The only exception was the optimum performance of the ISARIC 4C tool in predicting mortality among the Indian cohort which was domiciled from regions of relatively lower deprivation profiles compared to other ethnic minorities. Ethnic minorities presenting with pneumonia and low CURB65 scores (0-1) have higher mortality than Caucasians; Africans are at highest risk, followed by Caribbean, Indian and Pakistani. The findings in this study are consistent with those of a recent study of COVID-19 pneumonia patients ( $\mathrm{n}=279$ ) which found that, as a largely physiological assessment, CURB65 is an unreliable mortality risk tool in COVID-19 pneumonia. (19) Generally, ISARIC4C exhibits better performance among ethnic minorities than CURB65 which is likely to be in part due to its' inclusion of some risk factors to which ethnic minorities are predisposed: scoring > 2 comorbidities, CRP and oxygen saturations. The latter two assessment metrics (20) are typical of presentation with pneumonia. Ethnic minorities are more likely than Caucasians to present with multilobar pneumonia which this study finds is associated with admission from regions of highest air pollution deprivation, household overcrowding deprivation, housing quality deprivation, adult skills deprivation.

This study finds that air pollution deprivation, which is not currently incorporated in current severity or mortality risk stratification tools, increases the odds of presentation with radiological multilobar pneumonia and ITU admission among COVID-19 patients. Pollutants compromise the host's immune response against invading pathogens in the respiratory tract. (21) Chronic exposure to nitrogen dioxide and sulphur dioxide concentrations are associated with incidence of pneumonia (22) whilst particulate matter increases the activity of angiotensin-converting enzyme 2 receptors on cell surfaces (23), thus enhancing COVID-19 uptake by the lungs. In this study ethnic minorities apart from Chinese and Mixed were more likely to be admitted from regions of highest air pollution deprivation yet the risk of chronic exposure to air pollution is absent from current admission clinical risk tools. Minimising air pollution deprivation inequalities is essential in reducing the disease burden of community acquired pneumonia (24) (25) including COVID-19. 
Furthermore, this study identifies household overcrowding deprivation and poor housing quality, which are not currently included within severity or mortality risk stratification scores, as potential risk factors for presentation with radiological multilobar pneumonia and ITU admission among COVID-19 patients. The UK Biobank study has reported that patients with a COVID19 positive test were more likely to live in crowded households (26) and it is well established that patients admitted with non-COVID19 pneumonia are more likely to have been exposed to household overcrowding and housing quality failing to meet the Decent Homes Standard. (27) A national UK study found that living in a multigenerational household increased the risk of mortality from COVID-19 and this amounted to a 10-15\% elevated risk of COVID19 death among older females from South Asian background (28). Ethnic minorities are more likely to be hospitalised from the most overcrowded and poorest quality housing yet these risk factors are not incorporated within admission clinical risk tools. (24) Minimising housing inequality is essential for limiting the exposure to and spread of toxigenic species including bacteria, fungal and viral pathogens. (29)

Moreover, this study finds that adult skills deprivation including limited English language proficiency and low adult qualifications, which are not included within current severity or mortality risk stratification tools, are potential risk factors for presentation with radiological multilobar pneumonia and ITU admission. Coronaviruses cause pneumonia which gradually progresses to further lung zones between 2-14 days. (30) It is possible that the predominantly English language messaging may have contributed to later presentation in patients from regions of highest adult skills deprivation. In this study, Caribbean, Pakistani, African, Bangladeshi, Mixed and any other non-Caucasian ethnic subgroups were more likely than Caucasians to be admitted from regions of highest adult skills deprivation yet cultural differences and potentially lower English literacy which could reduce access to health to healthcare are not accounted for in current admission risk tools. Minimising education inequalities including by exploring strategies designed to widen access to and boost engagement with health messaging is essential for enhancing compliance with infection, prevention and control measures and ensuring timely presentation.

Whilst deprivation metrics are not included within current admission risk tools, the QCOVID tool (31) for predicting hospital admission incorporates the Townsend deprivation score which contains indicators for unemployment, household overcrowding, car and home ownership. However, a limitation of the Townsend score is the absence of air pollution data, housing quality data or adult skills data which are risk factors for presentation with multilobar pneumonia and ITU admission.

A surprising finding is that Caribbean patients did not appear to be at increased risk of mortality despite presenting 17 years older than African patients. This was despite both groups exhibiting a similarly high multimorbidity burden and being more likely than Caucasians to be admitted from regions of highest Wider BHS deprivation, Outdoor LE deprivation and Adult Skills deprivation. One of the challenges of analysing ethnic minority group data relates to small sample subgroups and wide confidence intervals which adds a level of uncertainty which may play a part. Nevertheless, several hypotheses have been put forward to explain the increased mortality among Africans including the high prevalence of Glucose-6-phosphate dehydrogenase(G6PD) deficiency, (32) which, it has been suggested, may increase viral replication and susceptibility to viral infections by inducing oxidative stress; antioxidants have been found to be protective against viral infection. Studies are needed to explore genetic, immunological and metabolic differences between African and Caribbean groups. (23) An important message is that individual ethnic minorities exhibit distinct risk factor profiles.

This study includes hospitalised COVID-19 patients within four hospitals across the West Midlands constituting one of the UK's largest NHS Trusts. It did not analyse COVID-19 patients who were not hospitalised or who died in the community.

\section{Conclusion}

Ethnic minorities exhibit younger age structures, higher multimorbidity and disproportionate exposure to unscored risk factors including obesity and deprivation resulting in potential triage to an inappropriate level of care with clinicians left falsely reassured regarding the severity of presentation and risk of deterioration. Household overcrowding deprivation, air pollution deprivation, housing quality deprivation and adult skills deprivation are associated with multilobar pneumonia on presentation and ITU admission. Risk tools need to reflect risk factors predominantly affecting ethnic minorities.

Consideration of multi-ethnic age structures, sex, Body Mass Index, $\mathrm{CCl}$ score, chest X-ray imaging and deprivation sub-indices on admission supports clinicians in stratifying high risk patients. COVID-19 admission clinical risk stratification tools need to be developed to account for risk factors to which ethnic minorities are predominantly exposed. This will enable the early identification of patients at risk of deterioration and ensure triage to an appropriate level of care.

Future studies need to relate these findings with populations from other urban rural regions with this level of granularity to inform national strategic planning on risk stratification and minimising health inequalities.

\section{List Of Abbreviations}

BAME- Black, Asian and Minority Ethnic group

BHS- Barriers to Housing and Services

CCl- Charlson Comorbidity Index (CCl)

CAP- Community Acquired Pneumonia

COVID-19-SARS-COV-2 virus infection

CKD- Chronic Kidney Disease 
EST- Education Skills and Training

Fig. - Figure

HRA-Health Research Authority

IHD- Ischaemic heart disease

IMD- Index of Multiple Deprivation Score

IQR- Interquartlie range

ITU- Intensive therapy unit (intensive care unit)

LE-Living Environment

PVD- Peripheral Vascular Disease

SD-Standard Deviation

T2DM-Type 2 Diabetes Mellitus

\section{Declarations}

\section{Ethics approval and consent to participate}

Ethical approval was obtained by the Health Research Authority: REC reference 21/HRA/1299. The study was registered by the UHB Research and Development department - RRK7305.

\section{Availability of data and materials}

All of the datasets used are presented within this paper.

\section{Competing interests}

The authors declare that they have no competing interests

\section{Funding}

No funding was received for this study

DT is funded by the MRC. MS is funded by the NIHR and reports grants from Astra Zeneca.

\section{Authors' contributions}

MS collected data, undertook data analysis, designed this study and wrote this paper. MS, BS, CRM, JV, DT, DD, WC made substantial contributions to the conception, design of the work and supported data interpretation. MS, BS, CRM, JV, DP, SL, DD, DT, WC revised the final manuscript. All authors contributed to and approved the final version of the manuscript.

\section{References}


1. ICNARC. Report on 2249 patients critically ill with COVID-19. [Online].; 2020 [cited 20205 10. Available from: https://www.icnarc.org/About/Latest-News Critically-III-With-Covid-19.

2. Smith S, et al.. Multidisciplinary research priorities for the COVID-19 pandemic. The Lancet Psychiatry. 2020 July; 7(7).

3. Wynants, L; et al. Prediction models for diagnosis and prognosis of covid-19 infection: systematic review and critical appraisal. BMJ. 2020 April;(DOI: 10

4. Gupta, R; et al. Systematic evaluation and external validation of 22 prognostic models among hospitalised adults with COVID-19: An observational cohc September.

5. Lim WS. Defining community acquired pneumonia severity on presentation to hospital: an international derivation and validation study. BMJ Thorax. $2 \mathrm{C}$

6. Gupta,R. ; et al. Development and validation of the ISARIC 4C Deterioration model for adults hospitalised with COVID-19: a prospective cohort study. The

7. UK Government. Age groups: GOV.UK. [Online].; 2020 [cited 20211 1. Available from: https://www.ethnicity-facts-figures.service.gov.uk/uk-population-by

8. Soltan M, Crowley L, Melville C, Varney J, Cassidy S, Mahida R, et al. To what extent are social determinants of health, including household overcrowdinc modulators of presentation, ITU admission and outcomes among patients with SARS-COV-2 infection in an urban catchment area i. BMC Public Health. 2020

9. Office for National Statistics. Deaths involving COVID-19 by local area and socioeconomic deprivation: deaths occurring between 1 March and 31 July 2 Available from:

https://www.ons.gov.uk/peoplepopulationandcommunity/birthsdeathsandmarriages/deaths/bulletins/deathsinvolvingcovid19bylocalareasanddeprivation/c

10. Department for Communities and Local Government. The English Index of Multiple Deprivation (IMD) 2015 - Guidance. [Online].; 2015 [cited 2019220. https://assets.publishing.service.gov.uk/government/uploads/system/uploads/attachment_data/file/464430/English_Index_of_Multiple_Deprivation_2015_.

11. Ministry of Housing,Communities \& Local Government. The English Indicies of Deprivation 2019. [Online].; 2020 [cited 2020 May 8. Available from: https://assets.publishing.service.gov.uk/government/uploads/system/uploads/attachment_data/file/833951/loD2019_Technical_Report.pdf.

12. GOV.UK. COVID-19: guidance for sampling and for diagnostic laboratories. [Online].; 2020 [cited 2020 December 10. Available from: https://www.gov.uk/ coronavirus-guidance-for-clinical-diagnostic-laboratories.

13. Charlson et al.. new method of classifying prognostic comorbidity in longitudinal studies: Development and validation. Journal of CLinical Epidemiology

14. Brtish Thoracic Society Guidelines for the Management of Community Acquired Pneumonia in Adults Update 2009 A Quick Reference Guide. United Kins 2023.

15. Knight Stephen; et al. Risk stratification of patients admitted to hospital with covid-19 using the ISARIC WHO Clinical Characterisation Protocol: developr BMJ. 2020 September;(doi:10.1136/bmj.m3339)

16. Zhu, Y; et al. Characteristics, service use, and mortality of clusters of multimorbid patients in England: a population-based study. The Lancet. 2019 Novel

17. Hui-Fang,Li; et al. Ethnic disparities in prevalence and clustering of cardiovascular disease risk factors in rural Southwest China. BMC Cardiovascular Dis

18. Whitty C. Rising to the challenge of multimorbidity. British Medical Journal. 2020 January.

19. Soltan M, Kim M. The ABCDE approach explained. BMJ. 2016 Oct 24; 355.

20. Nguyen Y, et al.. Applicability of the CURB-65 pneumonia severity score for outpatient treatment of COVID-19. J. Infect. 2020 Sept; 81.

21. Adaji $\mathrm{E}$, et al.. Understanding the effect of indoor air pollution on pneumonia in children under 5 in low- and middle-income countries: a systematic reviev December; 26(4)

22. Ji W, et al.. Prolonged effect of air pollution on pneumonia: a nationwide cohort study. European Respiratory Journal. 2017; DOI: 10.1183/1393003.cong

23. Pozzer A, et al.. Regional and global contributions of air pollution to risk of death from COVID-19. Cardiovascular Research. 2020 December; 116(14).

24. Fecht, D. et al.. Associations between air pollution and socioeconomic characteristics, ethnicity and age profile of neighbourhoods in England and the N $\epsilon$

25. Zanobetti A. Air Pollution and Pneumonia The "Old Man" Has a New "Friend". American Journal of Respiratory and Critical Care Medicine. 2010; 181(1).

26. Raisi-Estabragh Z. Greater risk of severe COVID-19 in Black, Asian and Minority Ethnic populations is not explained by cardiometabolic, socioeconomic c status: study of 1326 cases from the UK Biobank. Journal of Public Health. 2020 June; 42(3).

27. Maria Regina Alves Cardoso et al. Crowding: risk factor or protective factor for lower respiratory disease in young children?. BMC Public Health. 2004 Ju

28. Vahé Nafilyan et al. Ethnicity, Household Composition and COVID-19 Mortality: A National Linked Data Study. medRxiv. 2020.

29. Kuhn, D. et al.. Indoor Mold, Toxigenic Fungi, and Stachybotrys chartarum: Infectious Disease Perspective. Clin Microbiol Rev., https://www.ncbi.nlm.nih January; $16(1)$.

30. Prof David S Hui. Middle East respiratory syndrome coronavirus: risk factors and determinants of primary, household, and nosocomial transmission. The 18(8).

31. Clift A, et al.. Living risk prediction algorithm (QCOVID) for risk of hospital admission and mortality from coronavirus 19 in adults: national derivation an 321.

32. Y. Wu. Glucose-6-phosphate dehydrogenase deficiency enhances human coronavirus 229E infection. J Infect Dis. 2008 March.

\section{Figures}


Assessed for eligibility $(n=3671))$

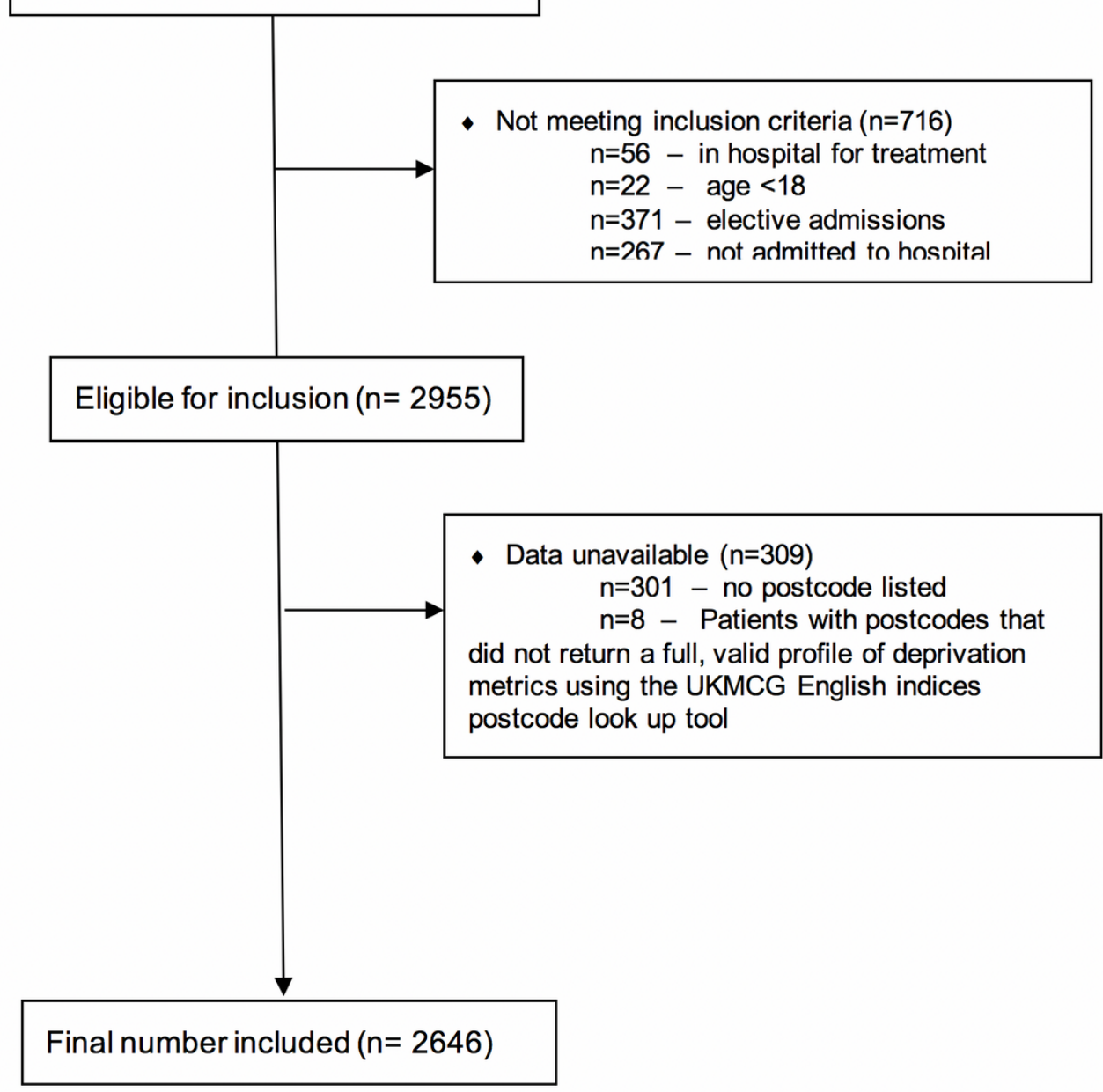

Figure 1

A CONSORT diagram showing participants assessed for eligibility, the inclusion criteria and the final number of participants included. 3671 consecutive patients were assessed for eligibility for inclusion into this study. 716 patients were excluded on account of having not met the inclusion criteria due to: ongoing hospitalisation on 1st September 2020(n=55), age <18 ( $n=22)$, attending hospital as an elective admission $(n=371)$ or attending hospital without $\operatorname{admission}(n=267)$. Patients eligible for inclusion in this study $(n=2955)$ were reviewed; patients without listed postcodes( $n=301)$ or postcodes not returning deprivation metrics $(n=8)$ could not be included in the analysed group $(n=2646)$. 

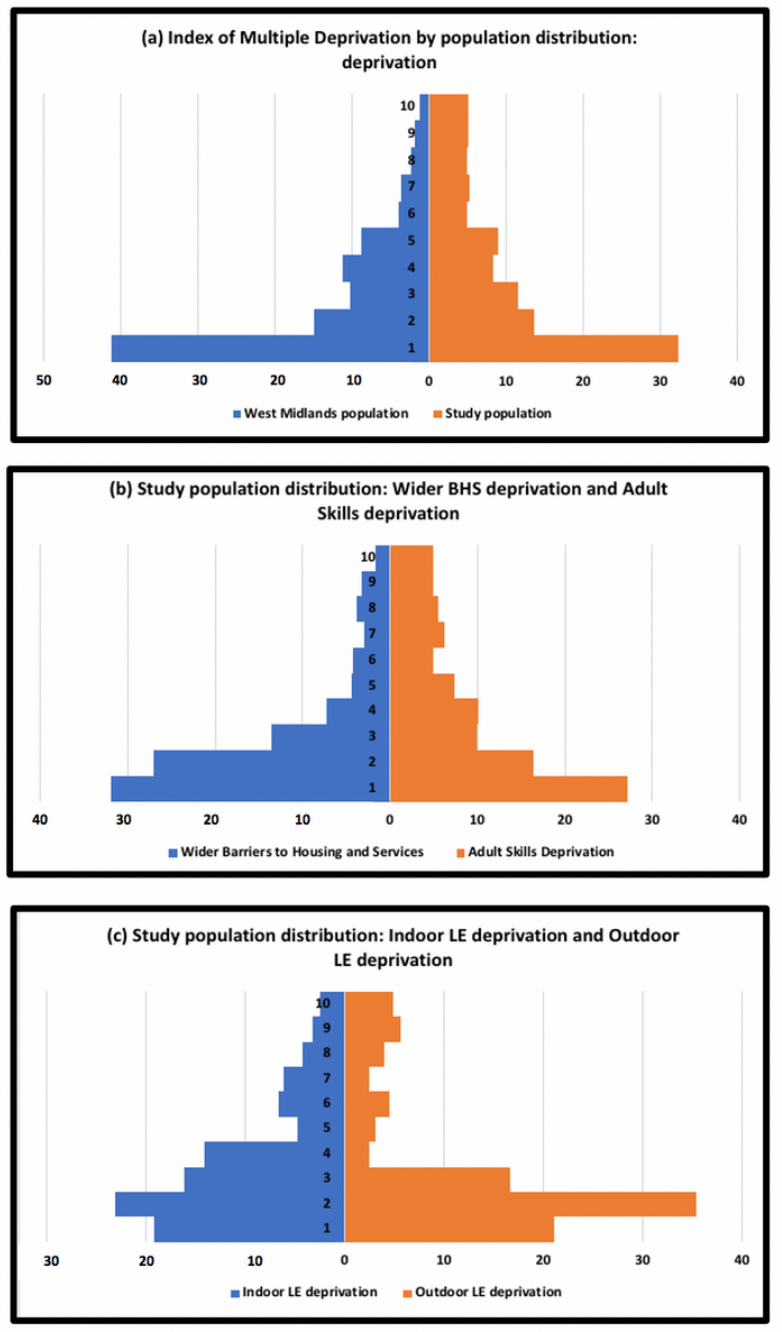

\section{Figure 2}

Population pyramid distributions of hospitalised COVID-19 positive patients: (a) Index of Multiple Deprivation (IMD) distribution in the West Midlands population in comparison with the study population, (b) Wider BHS deprivation sub-index distribution and adult skills deprivation sub-index distribution in the study population, (c) Indoor LE deprivation sub-index and Outdoor LE deprivation sub-index distribution in the study population. 

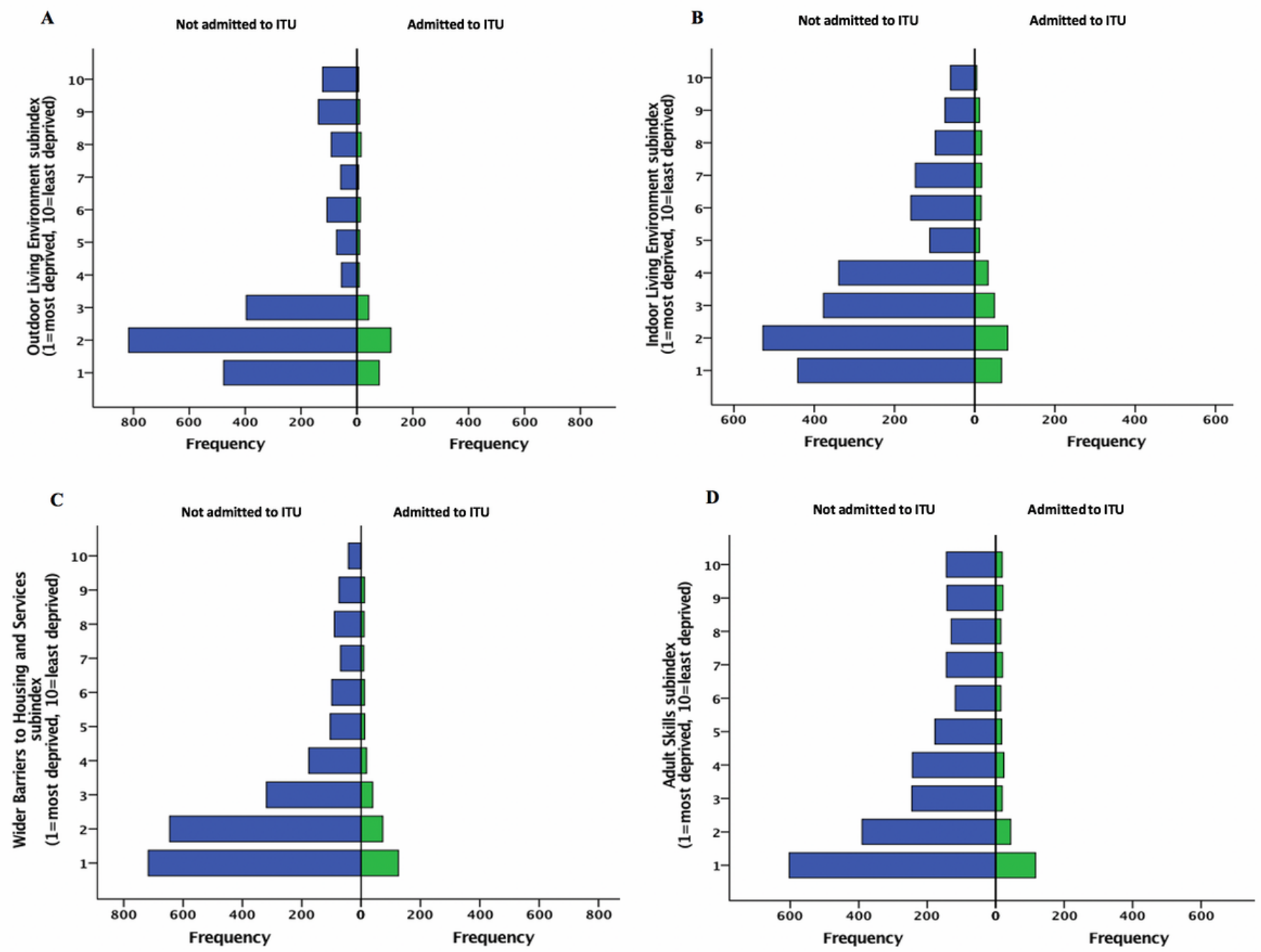

\section{Figure 3}

Population pyramid distributions of COVID-19 positive patients admitted to ITU from regions of (a) Outdoor Living Environment deprivation, (b) Indoor Living Environment deprivation, (c) Wider Barriers to Housing and Services deprivation, (d) Adult Skills deprivation 

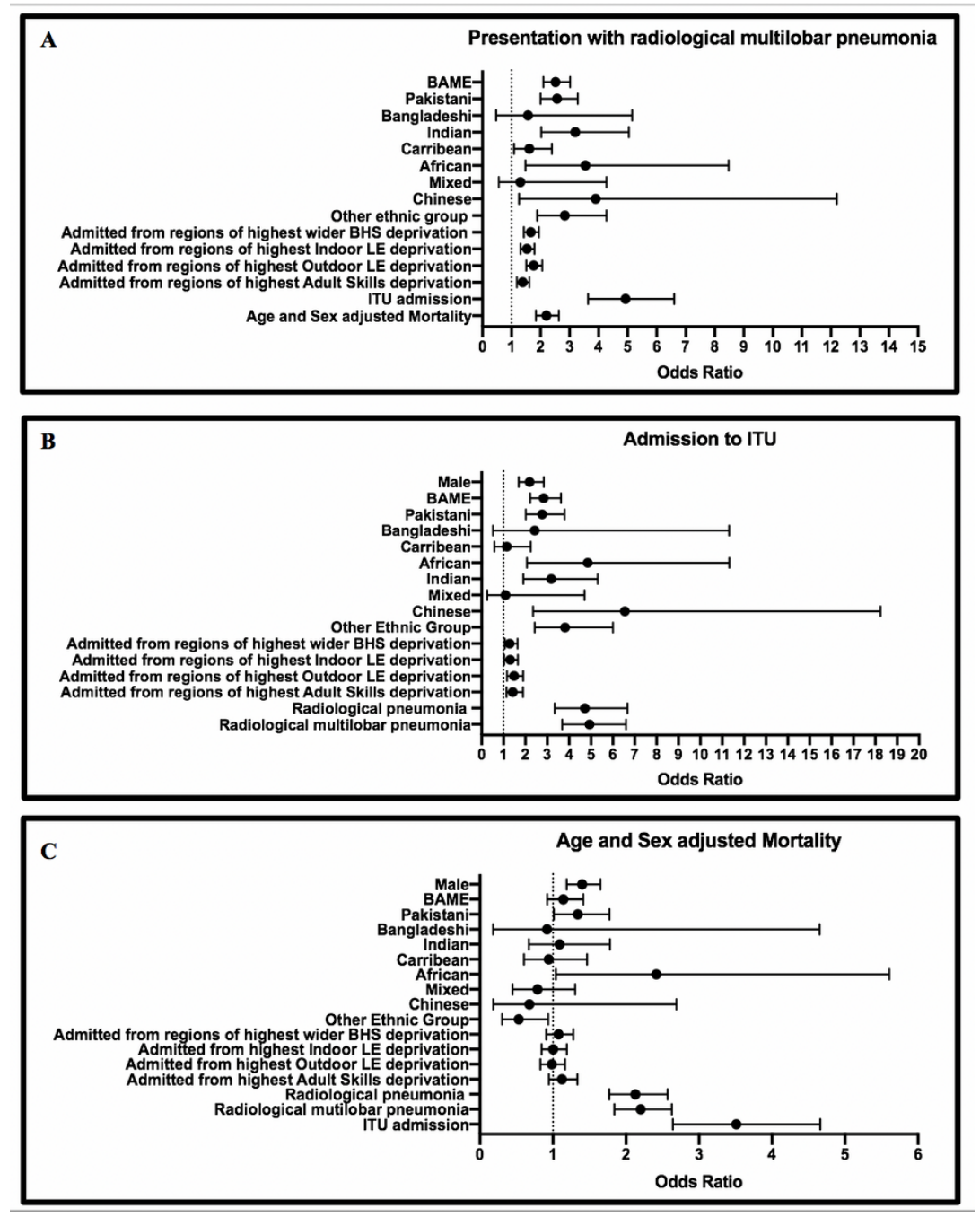

Figure 4

Odds ratios of hospitalised COVID-19 patients presenting with multilobar pneumonia, requiring ITU admission and mortality (age and sex adjusted) (a) Odds ratios of presentation with multilobar pneumonia by: gender, ethnicity (BAME, Pakistani, Bangladeshi, Indian, Caribbean, African, Mixed, Chinese, Other ethnic group vs. Caucasian), admission from sub-indices of highest deprivation (Wider BHS deprivation, Indoor LE deprivation, Outdoor LE deprivation, Adult Skills deprivation) vs. admission from all other deprivation sub-indices of the respective deprivation form, admission to ITU vs. not admitted to ITU and mortality (age and sex adjusted) vs. discharge. (b) Odds ratios of ITU admission by: gender, ethnicity (BAME, Pakistani, Bangladeshi, Indian, Caribbean, African, Mixed, Chinese, Other ethnic group vs. Caucasian), admission from sub-indices of highest deprivation (wider BHS deprivation, Indoor LE deprivation, Outdoor LE deprivation, Adult Skills deprivation) vs. admission from all other deprivation sub-indices of the respective deprivation form and presentation with pneumonia (radiological pneumonia vs. radiological multilobar pneumonia) vs. presentation without pneumonia; (c) Odds ratios of age and sex adjusted mortality by: gender, ethnicity (BAME, Pakistani, Bangladeshi, Indian, Caribbean, African, Mixed, Chinese, Other ethnic group vs. Caucasian), admission from sub-indices of highest deprivation (wider BHS deprivation, Indoor LE deprivation, Outdoor LE deprivation, Adult Skills deprivation) vs. admission from all other deprivation sub-indices of the respective deprivation form, presentation with pneumonia (radiological pneumonia, radiological multilobar pneumonia) vs. presentation without pneumonia and ITU admission vs. not admitted to ITU. 


\section{Comorbidities}

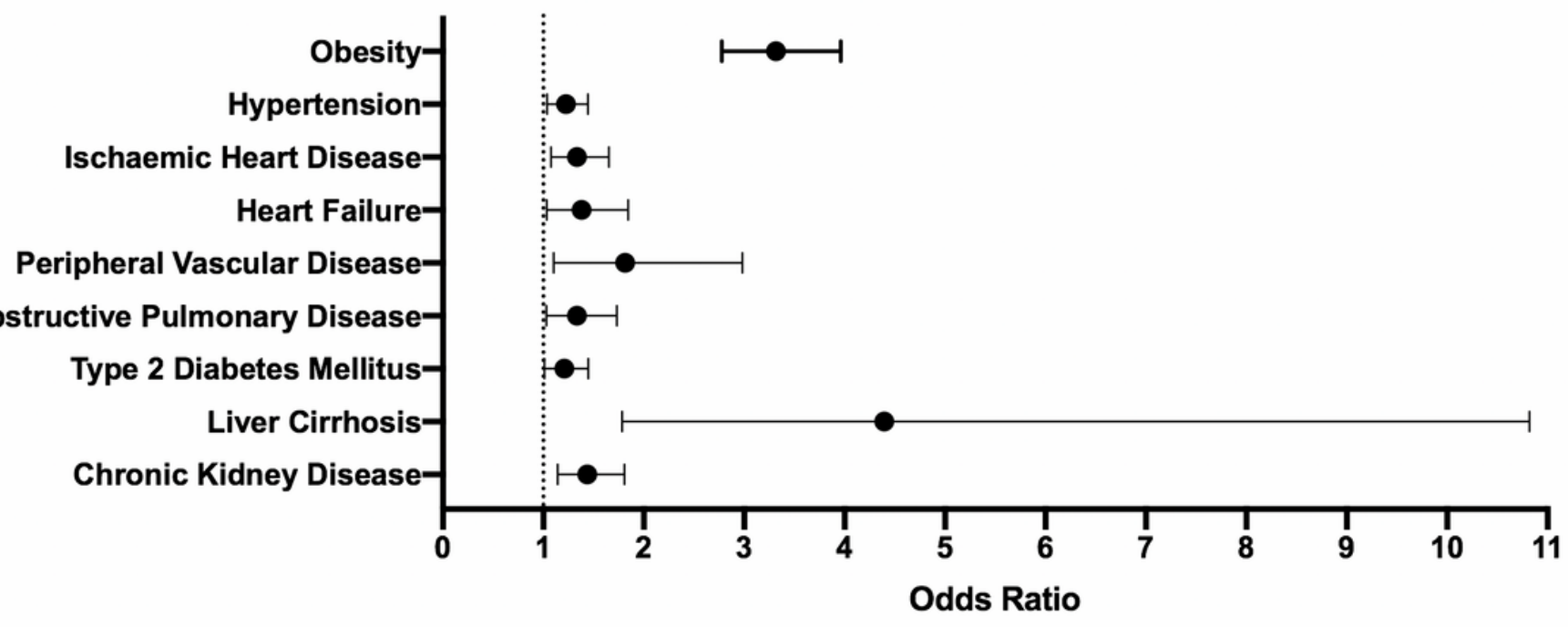

Figure 5

Odds ratios of mortality among COVID-19 patients by underlying obesity, hypertension, ischaemic heart disease, heart failure, peripheral vascular disease, COPD, type 2 diabetes mellitus, liver cirrhosis and chronic kidney disease
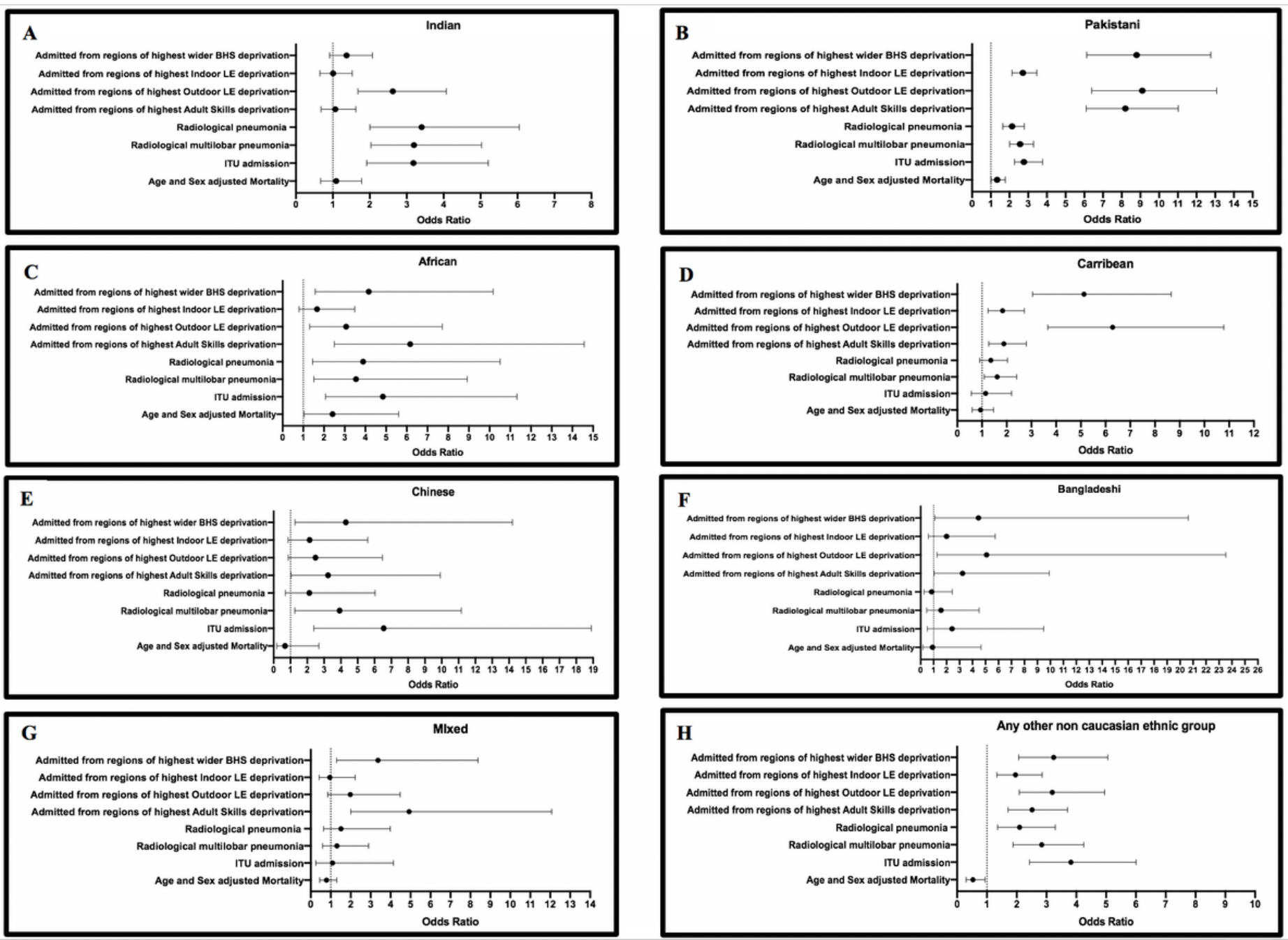

Figure 6 
Odds ratios of hospitalised COVID-19 positive patients of (a) Pakistani, (b) Indian, (c) Bangladeshi, (d) African, (e) Caribbean, (f) Chinese, (g) mixed and (h) any other ethnicity by: admission from sub-indices of highest deprivation (wider BHS deprivation, Indoor LE deprivation, Outdoor LE deprivation, Adult Skills deprivation), ITU admission and mortality (age and sex adjusted).

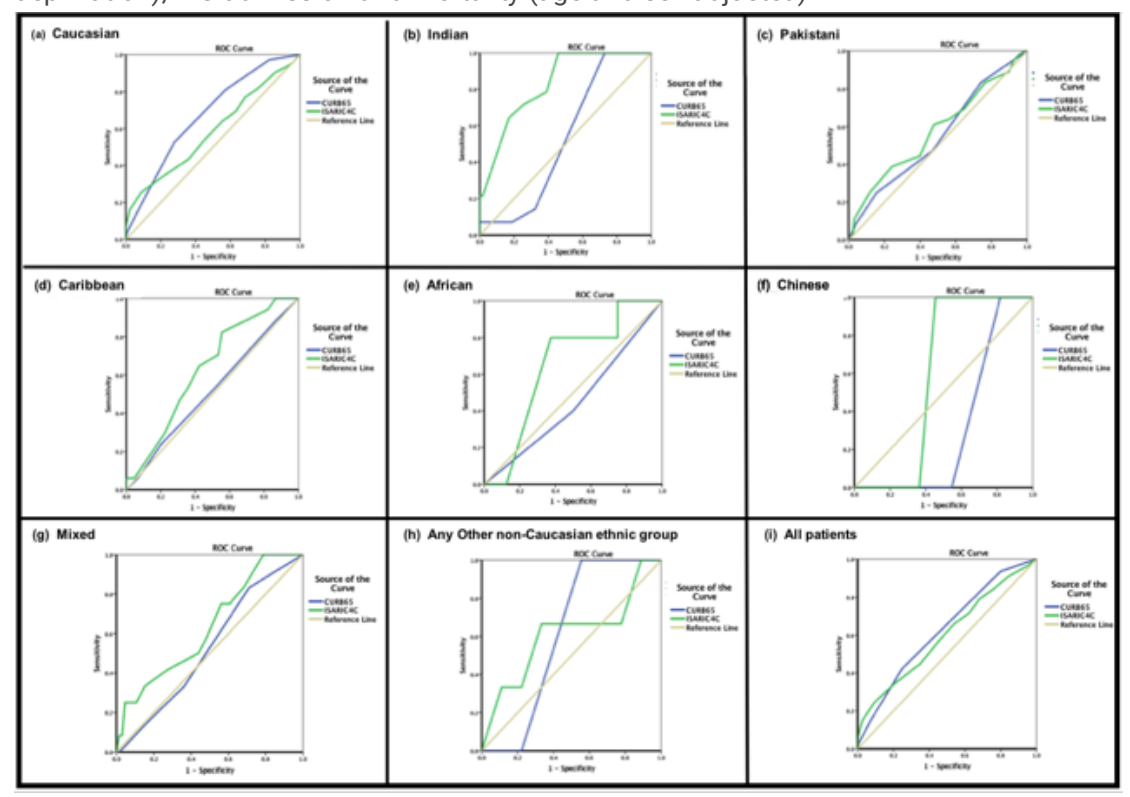

Figure 7

Graphs showing receiver operating characteristics (ROC) curve for the CURB65 and ISARIC 4C scores by ethnicity: (a) Caucasian, (b) Indian, (c) Pakistani, (d) Caribbean, (e) African, (f) Chinese, (g) Mixed, (h) any other ethnic group, (i) All patients

\section{Supplementary Files}

This is a list of supplementary files associated with this preprint. Click to download.

- Onlinesupplementnew.docx 